\title{
Synthetic bone replacement materials used for one-stage treatment of chronic osteomyelitis
}

\author{
A.V. Afanasyev, S.A. Bozhkova, V.A. Artyukh, D.V. Labutin, V.N. Liventsov, A.A. Kochish \\ Vreden National Medical Research Center of Traumatology and Orthopedics, Saint Petersburg, Russian Federation
}

\begin{abstract}
Introduction The paper presents a comparative analysis of one-stage treatment of chronic osteomyelitis using tobramycinimpregnated calcium sulfate and $\beta$-tricalcium phosphate mixed with antibiotics impregnated hydroxyapatite to fill in the cavitary defect of long bones. Material and methods The retrospective group (group 1, $\mathrm{n}=34$ ) was treated with tobramycin-impregnated calcium sulfate (Osteoset $\mathrm{T}$ ), and the prospective group (group 2, n $=25$ ) received $\beta$-tricalcium phosphate mixed with antibiotics impregnated hydroxyapatite (ReproBone). Results and discussion There were no significant differences between the groups of patients by gender, age, type of osteomyelitis and duration of the disease. The differences in the operating time, intraoperative blood loss and the size of the cavitary bone defect post debridement were not significant between the groups. There were no significant differences in preoperative level of CRP between the groups, with CRP being higher $(\mathrm{p}=0.02)$ in group 1 than in group 2, prior to the discharge from the hospital. Recurrence was seen in $35 \%(n=12)$ of group 1 and in $16 \%(n=4)$ of group 2 at a $21 / 2$-year follow-up. The risk of recurrence was found to be 2.2 times greater with use of commercially available biocomposite loaded with tobramycin that that with $\beta$-tricalcium phosphate mixed with hydroxyapatite (HR 2.206; CI 0.806-6.038) impregnated with antibiotics administered with preoperative microbiological findings. Conclusion Combined $\beta$-tricalcium phosphate based biocomposites mixed with hydroxyapatite impregnated with broad-spectrum antibiotics showed greater efficacy for methicillin-resistant staphylococcus aureus in the treatment of chronic osteomyelitis filling cavitary bone defect of long bones as compared to tobramycin-impregnated calcium sulfate.
\end{abstract}

Keywords: osteomyelitis, bone replacement material, cavitary bone defect

\section{INTRODUCTION}

Osteomyelitis is one of the most severe surgical complications of trauma and orthopedic patients. A chronic disease is characterized by the formation of sequesters and numerous bacterial species forming biofilms [1]. Surgical treatment of chronic osteomyelitis results in recurrence in $20-30 \%$ of cases and limb amputation in $16.75 \%$ [2-4]. Radical debridement with biofilms removal invariably leads to significant defects in the bone and soft tissue that must be ultimately addressed to replace the segmental bone defect and regain limb function.

The two-stage replacement of bone defects is currently the most common method with use of an antibacterial polymethylmethacrylate (PMMA) spacer placed at the first stage after debridement. However, the bone cavity filled with antibioticloaded PMMA cement requires a repeated surgical intervention for removal and bone grafting and leads to increased duration of treatment [5], an additional stress on the patient and greater financial costs of treatment, and the method cannot be considered optimal for the replacement of bone defects. Different synthetic bioresorbable composite material (calcium sulfate, $\beta-3$ calcium phosphate, hydroxyapatite, etc.) impregnated with antibiotics can be used to fill in the bone defect after surgical debridement of the infection site during one-stage treatment $[6,7]$. One of the advantages of these materials is that their implantation ensures the local delivery of a high concentration of antibiotics being simultaneously involved in the bone regeneration [8]. The antibiotic is known to be released from resorbed materials and penetrate the tissues as a $100 \%$ substance, and PMMA cements can release no more than $25 \%$ of the antibiotic $[9,10]$. Commercially available synthetic officinal biocomposite materials of calcium sulfate containing tobramycin (for example, Osteoset T) have been long used for the treatment of osteomyelitis. Major disadvantages of this material in clinical practice included rapid resorption and prolonged postoperative wound discharge $[8,11]$. Positive experimental results of beta-3 calcium phosphate with hydroxyapatite (ReproBone, additionally impregnated with an antibiotic [12] used for the treatment of chronic osteomyelitis have provided sufficient evidence for the material considered effective as a medical treatment.

The objective was to evaluate the clinical efficacy of single-stage treatment of chronic osteomyelitis with the use of a biocomposite material $\beta$-3-calcium 
phosphate-hydroxyapatite (3CP-HA), additionally ex tempore impregnated with an antibiotic, in comparison with the use of calcium sulfate (CS) based officinal material with tobramycin.

\section{MATERIAL AND METHODS}

The interventional study included 59 patients who were assigned to two groups. The retrospective group (Group I) included 34 patients who underwent singlestage treatment of chronic osteomyelitis with bone cavities filled with CS with tobramycin (Osteoset T) between 2010 and 2014. The prospective group (Group II) included 25 patients who underwent comprehensive treatment of chronic osteomyelitis with bone defects filled with 3CP-HA (ReproBone) and additional intraoperative impregnation with an antibiotic (vancomycin $2 \mathrm{~g}$ or fosfomycin $4 \mathrm{~g}$, or cefuroxime $1.5 \mathrm{~g}$ per 20 grams of material), depending on bacterial culture tests of biomaterial samples collected from the infection site (aspirate, tissue biopsy) or empirically in their absence between 2015 and 2018. Inclusion criteria were chronic osteomyelitis of long bones 3 and 4 anatomic types III and IV, physiological classes A and $\mathrm{B}$ as grouped by the Cierny-Mader classification, replacement of a cavity bone defect, satisfactory condition of soft tissues for adequate closure of the surgical wound requiring no soft tissue coverage, the possibility of long-term (at least 6-8 weeks) therapy including oral AB-therapy, no signs of exacerbation of chronic osteomyelitis including clinical manifestations (infiltration, pronounced edema, soft tissue hyperemia, local hyperthermia, infectious inflammation of the subcutaneous tissues, fasciitis, soft tissue leaks) and/ or systemic inflammatory response syndrome in the form of a combination of two or more clinical and laboratory signs: temperature $>38^{\circ} \mathrm{C}$ or $<36^{\circ} \mathrm{C}$, heart rate $>90$ per minute, respiratory rate $>20$ per minute or hyperventilation $(\mathrm{PaCO} 2<32 \mathrm{mmHg}$ ), white blood cells count $>12 \times 10^{9} / \mathrm{L}$ or $<4 \times 10^{9} / \mathrm{L}$ or neocytosis $>10 \%$, absence of non-debrided foci of infection of different localization (trophic ulcers, urogenital infection, oral infection and others).

There were $93 \%(n=55)$ and $7 \%(n=4)$ cases of osteomyelitis classified as the Cierny-Mader anatomical types 3 and 4, respectively, and $76 \%$ and $24 \%$ as physiological classes B and A. Impaired supporting ability of the limb in anatomic type $4(n=4)$ was associated with delayed fracture healing due to infection resulting from metal construct fixation. All patients with anatomical type 4 underwent additional external fixation of the involved segment. Osteomyelitis location was in the tibia $(76 \% ; n=45)$, femur $(14 \% ; n=8)$ and in the humerus $(10 \% ; n=10)$. the disease was diagnosed as postoperative $(47 \%, \mathrm{n}=28)$, posttraumatic $(39 \%$; $\mathrm{n}=23)$, hematogeneous $(14 \% ; \mathrm{n}=8)$. The condition was recurrent in $78 \%(n=46)$.

The average age of patients was 47 years (CI $95 \%$, range $42-52$ years) in the retrospective group and 39 years (CI95\%, range $34-44$ years) in the prospective group (Table 1). All patients underwent surgical debridement, fenestrated osteotrepanation for the purpose of thorough osteonecrectomy of the infection site to reach a healthy bone (blood dew symptom) followed by copious wound irrigation with antiseptic solutions (3\% hydrogen peroxide, $0.05 \%$ chlorhexidine, $0.1-0.2 \%$ lavasept) and saline solution. Bone defect size, operating time, intraoperative blood loss, WBC count and CRP level were evaluated preoperatively and at 10-12 days post surgery identifying the type of pathogen and infection eradication. The infection was considered arrested with no postoperative signs of inflammation for at least a year (fever, edema, hyperemia, severe pain, prolonged (more than 10 days) discharge from the postoperative wound, leukocytosis, fistulas). The mean follow-up period was 43 months (MCI 31-48) in the basic sample, 48 months (MCI 36.5-52.5) in Group I and 34 months (MCI 27-46) in Group II. Statistical data analysis was performed using the GraphPad Prism 6.0 program (USA). The patient's age was presented as the mean with confidence intervals of $95 \%$ (CI $95 \%$ ), and other quantitative variables presented as the median with a $25-75 \%$ interquartile interval (MCI 25-75\%). Non-parametric Mann-Whitney test was used to look at significant differences. Qualitative data was presented in the form of the number of events, and the Fisher test was used to assess the significance of differences between the groups. A value of $\mathrm{P}<0.05$ was considered statistically significant.

\section{RESULTS}

There were no significant differences between the groups by sex, age, type of osteomyelitis, and duration of the disease. Complete fracture consolidation was achieved in all patients with the Cierny-Mader anatomical type 4 . There were insignificant differences in the operating time, intraoperative blood loss and the volume of the cavitary bone defect size after debridement between groups of comparison (Table 1). 
Table 1

Characterization of the groups of comparison

\begin{tabular}{|l|c|c|c|}
\hline \multicolumn{1}{|c|}{ Description } & Group I, $\mathrm{n}=34$ & Group II, $\mathrm{n}=25$ & P value \\
\hline Number of patients & 34 & 25 & \\
\hline Recurrence, $\mathrm{n}(\%)$ & $12(35)$ & $4(16)$ & 0.6 \\
\hline Preoperative WBC count, $10^{9} / \mathrm{L}, \mathrm{Me}(\mathrm{MCI})$ & $6.9(5.7-8.6)$ & $6.4(5.6-8.6)$ & 0.41 \\
\hline WBC count prior to discharge, $10^{9} / \mathrm{L}, \mathrm{Me}(\mathrm{MCI})$ & $6.9(5.1-8.3)$ & $6.2(5.3-7.8)$ & 0.12 \\
\hline Preoperative CRP, $\mathrm{mg} / \mathrm{mL}, \mathrm{Me}(\mathrm{MCI})$ & $5.4(2.2-9.8)$ & $2.8(0.78-5.6)$ & 0.02 \\
\hline CRP prior to discharge, Mr/Mл, Me (MCI) & $7.1(3.1-16)$ & $3.9(2.1-6.5)$ & 0.13 \\
\hline Bone defect size, $\mathrm{mL}, \mathrm{Me}(\mathrm{MCI})$ & $7(4-12)$ & $10(5.5-20)$ & 0.7 \\
\hline Blood loss, mL, Me (MCI) & $100(88-263)$ & $150(50-350)$ & 0.35 \\
\hline Operating time, min., Me (MCI) & $85(64-136)$ & $95(75-155)$ & 0.89 \\
\hline Duration of the disease, years, Me (MCI) & $2(1-5.3)$ & $2(1-4)$ & 0.1 \\
\hline Polymicrobe etiology, \% (n) & $11.7(4)$ & $32(8)$ & \\
\hline
\end{tabular}

The white blood cells count was comparable in both groups throughout the entire period of hospitalization. Preoperative CRP level did not significantly differ between the groups and was significantly lower in 3CS-HA patients than in CS and tobramycin group prior to the discharge from the hospital. Local antibacterial therapy as a component of bone substitution material was produced with cefuroxime in $56 \%$, vancomycin in $36 \%$, and fosfomycin in $8 \%$ of patients of Group II. Bacterial culture tests were performed for all patients of the prospective group and in $82 \%$ of the retrospective group. Positive microbial growth was seen in 71.4 and $92 \%$ of cases of the retrospective and prospective groups, respectively. Chronic osteomyelitis was caused by microbial associations of two or more microorganisms in $17.8 \%$ (5 out of 28 ) and $32 \%$ ( 8 out of 25) cases, in Groups I and II, respectively, including gram-negative bacteria in 1 of 5 and in 3 out of 8 cases, respectively. The leading causative agents of osteomyelitis in both groups were staphylococci ( $S$. aureus, S. epidermidis and other coagulase-negative staphylococci) with $71 \%$ and $66 \%$ in Groups I and II, respectively (Table 2).

Table 2

Etiology of infections in the groups of comparison, \% (n)

\begin{tabular}{|l|c|c|c|}
\hline \multicolumn{1}{|c|}{ Etiology of infections } & Group I, \% (n) & Group II, \% (n) & p value \\
\hline Total & $\mathbf{4 4}(\mathbf{1 5})$ & $\mathbf{6 0}(\mathbf{1 5})$ & $\mathbf{0 . 5 3}$ \\
\hline Involving Gram-positrive (+) & $41(14)$ & $60(15)$ & 1.0 \\
\hline Involving Gram-negative (-) & $3(1)$ & 0 & $\mathbf{0 . 5 3}$ \\
\hline \multicolumn{3}{|c|}{ Microbial associations } \\
\hline Total & $\mathbf{1 5 ( 5 )}$ & $\mathbf{3 2}(\mathbf{8})$ & 0.21 \\
\hline involving MRS* & $12(4)$ & $4(1)$ & 0.08 \\
\hline Involving Gram-negative (-) & $3(1)$ & $8(2)$ & 0 \\
\hline No growth & $23(8)$ & $\mathbf{2 5}$ & \\
\hline Samples not collected & $18(6)$ & $\mathbf{3 4}$ & \\
\hline Total & &
\end{tabular}

*MRS, methicillin-resistant staphylococci (MRSA+MRSE).

Table 3

Spectrum of pathogens in the groups of comparison

\begin{tabular}{|c|c|c|c|c|c|}
\hline \multirow{2}{*}{ Pathogens } & \multicolumn{2}{|c|}{ Group I } & \multicolumn{2}{|c|}{ Group II } & \multirow{2}{*}{ p value } \\
\hline & abs. number & $\%$ & abs. number & $\%$ & \\
\hline Staphylococcus aureus & 14 & 52 & 14 & 40 & 0.44 \\
\hline $\begin{array}{l}\text { Staphylococcus epidermidis } \\
\text { and other CNS* }\end{array}$ & 5 & 19 & 9 & 26 & 0.56 \\
\hline $\begin{array}{l}\text { Gram(-) bacteria (fam. } \\
\text { Enterobacteriaceae and } \\
\text { Pseudomonas aeruginosa) }\end{array}$ & 2 & 7 & 3 & 8 & 1.0 \\
\hline Others & 6 & 22 & 9 & 26 & 1.0 \\
\hline Total & 27 & 100 & 35 & 100 & \\
\hline
\end{tabular}

*CNS, coagulase-negative staphylococci. 
Recurrent infection developed in 12 patients (35\%) of Group I within the first postoperative year in $67 \%$ and later in $33 \%$ of cases (Table 4). The pathogens identified in the patients were staphylococci $(\mathrm{n}=7 ; 58 \%)$ including methicillin-resistant strains $(n=2)$; enterococcus $(n=1 ; 8.5 \%)$ and a microbial association involving the methicillin-resistant strain of S. epidermidis $(\mathrm{n}=1 ; 8.5 \%)$. The pathogen was not identified in 1 patient $(8.5 \%)$ with no intraoperative culture available, and no growth was seen in 2 cases $(16.5 \%)$. Group II showed recurrence in 4 patients (16\%) including 3 cases who developed re-infection in the first 6 postoperative months $(\mathrm{n}=3)$ and a year later $(\mathrm{n}=1)$ (Table 4). The recurrence was caused by microbial associations $(\mathrm{n}=2 ; 50 \%)$, Staphylococcus aureus $(\mathrm{n}=2 ; 50 \%)$ including a methicillin-resistant strain $(n=1)$. The patients were locally treated with vancomycin $(n=3)$, cefuroxime $(n=1)$, and local antibacterial therapy appeared to be ineffective $(n=2)$ in a patient with $E$. coli who used vancomycin as part of the microbial association, and another patient with MRSA who was treated with cefuroxime.

Table 4

Recurrence seen in the groups

\begin{tabular}{|l|c|c|}
\hline \multicolumn{1}{|c|}{ Recurrence } & Group I, \% (n) & Group II, \% (n) \\
\hline Under 1 month & $16.7(2)$ & $25(1)$ \\
\hline $1-12$ months & $50(6)$ & $50(2)$ \\
\hline$>12$ months & $33.3(4)$ & $25(1)$ \\
\hline Total & $100(12)$ & $100(4)$ \\
\hline
\end{tabular}

Stable remission of osteomyelitis was achieved in $65 \%$ (22 of 34) patients in Group I with a mean follow-up period of 48 months (MCI 36.5-52.5), and in $84 \%$ ( 21 of 25 ) patients of Group II with a mean follow-up period of 34 months (MCI 27-46). Thus, the risk of recurrence was more than 2 times greater with the use of an officinal biocomposite material than that with 3CP-HA (HR 2.206 with CI 0.806-6.038) impregnated with an antibiotic.

\section{DISCUSSION}

The effectiveness of CS with tobramycin used as part of the comprehensive treatment of chronic osteomyelitis in our series was $65 \%$ at a 2 -year followup, which is inferior to the reports showing arrest of infection in $80 \%$ of similar cohorts of patients [13]. The differences can be ascribed to 3 patients who showed a discharge of more than 14 days and were included in the recurrence subgroup who underwent re-debridement. However, many researchers do not attribute similar complications to relapses of the infectious process. G. Humm et al. [11] reviewed the outcomes of 21 patients treated with Osteoset $\mathrm{T}$ for osteomyelitis of the tibia and encountered a wound discharge in the early postoperative period in $33 \%$ cases and delayed wound healing in $52 \%$. J.Y. Ferguson et al. [14 reported their experience using a CS antibiotic carrier containing tobramycin and prolonged wound ooze as self-limiting with the infection being resolved in $90.8 \%$ cases at follow-up and the wound associated with $75.4 \%$ healed with no oozing or collection of fluid. Prolonged wound ooze can be caused by quick dissolution of CS (3 to 12 weeks) as compared to 3CS-HA (6 to 12 months) and hydroxyapatite ( up to 12 years) [15]. The resorption of CS is reported to result in an acidic medium which can lead to prolonged postoperative wound discharge and problematic healing of the postoperative wound [8]. CS is known as an osteoconductive material with no osteoinductive and osteogenic properties [16] which are not characteristic of 3KF-HA. The two materials included in CS-HA are characterized by osteoconductivity, biocompatibility, low allergenic potential and can stimulate the growth and remodeling of new bone [17, 18].

Antibiotic loaded 3CS-HA used for filling in cavitarybone defects oflong bones in ourseries allowed us to increase the effectiveness of the treatment of chronic osteomyelitis by $84 \%$. Failures ( 2 of 4 cases) in our series were caused by a polyfocal involvement of the segment with the recurrence developed at one nidus of bone destruction. Recurrence developed in $66.6 \%$ patients of the retrospective group and was caused by staphylococci including methicillinresistant strains in $25 \%$, and tobramycin in the Osteoset-T failed to provide an efficient antimicrobial therapy. Microbial associations with gram(-) bacteria were cultured in three patients of Group II. In two cases, the bone substitution material was impregnated with a broad - spectrum drug, fosfomycin, which showed the effectiveness in experimental studies [12] and in clinical use [19] and could provide efficient infection eradication. With systemic antimicrobial therapy with broad-spectrum drugs, vancomycin used locally in comprehensive treatment of osteomyelitis caused by Staphylococcus aureus associated with E. coli did not allow infection eradication in one case. The acute phase of osteomyelitis could have caused the recurrence of infection at the time of surgery (preoperative WBC $16.7 \times 10^{9}$; CRP, $57.7 \mathrm{mg} / \mathrm{mL}$ ), and the patient developed the recurrence in the early postoperative period.

\section{CONCLUSION}

The one-stage surgical treatment is a preferred option for the patient, the attending physician and for the health care system. Combined $\beta$-tricalcium phosphate based biocomposites mixed with hydroxyapatite impregnated with broad-spectrum antibiotics showed greater efficacy for methicillin- 
resistant staphylococcus aureus in the treatment of long bones as compared to tobramycin-impregnated chronic osteomyelitis filling cavitary bone defect of calcium sulfate.

Funding The authors received no financial support for the research and/or authorship of this article.

Declaration of Conflicting Interests The authors declared no potential conflicts of interest with respect to the research, authorship, and/or publication of this article.

\section{REFERENCES}

1. Brady R.A., Leid J.G., Calhoun J.H., Costerton J.W., Shirtliff M.E. Osteomyelitis and the role of biofilms in chronic infection. FRMS Immunol. Med. Microbiol., 2008, vol. 52, no. 1, pp. 13-22. DOI: 10.1111/j.1574-695X.2007.00357.x

2. Conterno L.O., da Silva Filho C.R. Antibiotics for treating chronic osteomyelitis in adults. Cochrane Database Syst. Rev., 2009, no. 3, pp. CD004439. DOI: 10.1002/14651858.CD004439.pub2

3. Jiang N., Ma Y.F., Jiang Y., Zhao X.Q., Xie G.P., Hu Y.J., Qin C.H., Yu B. Clinical Characteristics and Treatment of Extremity Chronic Osteomyelitis in Southern China: A retrospective analysis of 394 consecutive patients. Medicine (Baltimore), 2015, vol. 94, no. 42, pp. e1874. DOI: 10.1097/ MD.0000000000001874

4. Tulner S.A.F., Schaap G.R., Strackee S.D., Besselaar P.P., Luitse J.S.K., Marti R.K. Long-term results of multiple-stage treatment for posttraumatic osteomyelitis of the tibia. J. Trauma Inj. Infect. Crit. Care, 2004, vol. 56, no. 3, pp. 633-642.

5. Dimitriou R., Mataliotakis G.I., Angoules A.G., Kanakaris N.K., Giannoudis P.V. Complications following autologous bone graft harvesting from the iliac crest and using the RIA: A systematic review. Injury, 2011, vol. 42, no. Suppl. 2, pp. S3-S15. DOI: 10.1016/j.injury.2011.06.015

6. Mäkinen T.J., Veiranto M., Lankinen P., Moritz N., Jalava J., Törmälä P., Aro H.T. In vitro and in vivo release of ciprofloxacin from osteoconductive bone defect filler. J. Antimicrob. Chemother., 2005, vol. 56, no. 6, pp. 1063-1068. DOI: 10.1093/jac/dki366

7. Fernandez de Grado G., Keller L., Idoux-Gillet Y., Wagner Q., Musset A.M., Benkirane-Jessel N., Bornert F., Offner D. Bone substitutes: a review of their characteristics, clinical use, and perspectives for large bone defects management. J. Tissue Eng., 2018, vol. 9, pp. 2041731418776819. DOI: $10.1177 / 2041731418776819$

8. McLaren A.C. Alternative materials to acrylic bone cement for delivery of depot antibiotics in orthopaedic infections. Clin. Orthop. Relat. Res., 2004, no. 427, pp. 101-106. DOI: 10.1097/01.blo.0000143554.56897.26

9. Privolnev V.V., Rodin A.V., Karakulina E.V. Mestnoe primenenie antibiotikov v lechenii infektsii kostnoi tkani [Local use of antibiotics in the treatment of bone tissue infections]. Klinicheskaia Mikrobiologiia i Antimikrobnaia Khimioterapiia, 2012, no. 2, pp. 118-131. (in Russian)

10. Uskoković V., Hoover C., Vukomanović M., Uskoković D.P., Desai T.A. Osteogenic and antimicrobial nanoparticulate calcium phosphate and poly(D,L-lactide-co-glycolide) powders for the treatment of osteomyelitis. Mater. Sci. Eng. C. Mater. Biol. Appl., 2013, vol. 33, no. 6, pp. $3362-3373$. DOI: $10.1016 /$ j.msec.2013.04.023

11. Humm G., Noor S., Bridgeman P., David M., Bose D. Adjuvant treatment of chronic osteomyelitis of the tibia following exogenous trauma using OSTEOSET(®)-T: a review of 21 patients in a regional trauma centre. Strategies Trauma Limb Reconstr., 2014, vol. 9, no. 3, pp. 157-161. DOI: $10.1007 / \mathrm{s} 11751-014-0206-\mathrm{y}$

12. Konev V.A., Bozhkova S.A., Netylko G.I., Afanasev A.V., Rumakin V.P., Poliakova E.M., Rukina A.N., Parfeev D.G. Rezultaty primeneniia fosfomitsina dlia impregnatsii osteozameshchaiushchikh materialov pri lechenii khronicheskogo osteomielita [The results of phosphomycinum using for impregnation of bone substitute materials during chronic osteomyelitis treatment]. Travmatologiia i Ortopediia Rossii, 2016, vol. 22, no. 2, pp. 43-56. (in Russian)

13. Chang W., Colangeli M., Colangeli S., Di Bella C., Gozzi E., Donati D. Adult osteomyelitis: debridement versus debridement plus Osteoset T pellets. Acta Orthop. Belg., 2007, vol. 73, no. 2, pp. 238-243.

14. Ferguson J.Y., Dudareva M., Riley N.D., Stubbs D., Atkins B.L., McNally M.A. The use of a biodegradable antibiotic-loaded calcium sulphate carrier containing tobramycin for the treatment of chronic osteomyelitis: a series of 195 cases. Bone Joint J., 2014, vol. 96-B, no. 6, pp. 829-836. DOI: $10.1302 / 0301-620 X .96 \mathrm{~B} 6.32756$

15. Ferguson J., Diefenbeck M., McNally M. Ceramic Biocomposites as Biodegradable Antibiotic Carriers in the Treatment of Bone Infections. J. Bone Jt. Infect., 2017, vol. 2, no. 1, pp. 38-51. DOI: 10.7150/jbji.17234.

16. Beuerlein M.J., McKee M.D. Calcium sulfates: what is the evidence? J. Orthop. Trauma, 2010, vol. 24, no. Suppl. 1, pp. S46-S51. DOI: 10.1097/ BOT.0b013e3181cec48e

17. Galois L., Mainard D. Bone ingrowth into two porous ceramics with different pore sizes: an experimental study. Acta Orthop. Belg., 2004, vol. 70, no. 6, pp. 598-603.

18. Bansal R., Patil S., Chaubey K.K., Thakur R.K., Goyel P. Clinical evaluation of hydroxyapatite and $\beta$-tricalcium phosphate composite graft in the treatment of intrabony periodontal defect: a clinico-radiographic study. J. Indian Soc. Periodontol., 2014, vol. 18, no. 5, pp. 610-617. DOI: 10.4103/0972-124X.142455

19. Bozhkova S.A., Kasimova A.R., Borisov A.M., Artiukh V.A., Liventsov V.N. Kliniko-ekonomicheskaia effektivnost ispolzovaniia fosfomitsina i vankomitsina dlia impregnatsii speiserov pri khirurgicheskom lechenii patsientov s periproteznoi infektsiei [Clinical-and-economical efficiency of using phosphomycinum and vancomycinum for impregnation of spacers in surgical treatment of patients with periprosthetic infection]. Zabaikalskii Meditsinskii Vestnik, 2017, no. 2, pp. 122-131. (in Russian)

Received: 27.05 .2020

\section{Information about the authors:}

1. Aleksandr V. Afanasyev, M.D.,

Vreden National Medical Research Center of Traumatology and Orthopedics, Saint Petersburg, Russian Federation

2. Svetlana A. Bozhkova, M.D., Ph.D.,

Vreden National Medical Research Center of Traumatology and Orthopedics, Saint Petersburg, Russian Federation

3. Vasilii A. Artyukh, M.D., Ph.D.,

Vreden National Medical Research Center of Traumatology and Orthopedics, Saint Petersburg, Russian Federation, Email: vaartjuh@win.rniito.ru

4. Dmitry V. Labutin, M.D.,

Vreden National Medical Research Center of Traumatology and Orthopedics, Saint Petersburg, Russian Federation

5. Vitaly N. Liventsov, M.D.,

Vreden National Medical Research Center of Traumatology and Orthopedics, Saint Petersburg, Russian Federation

6. Andrew A. Kochish, M.D.

Vreden National Medical Research Center of Traumatology and Orthopedics, Saint Petersburg, Russian Federation, Email: aakochish@rniito.ru 